\title{
Successful Intensive Lipid-lowering Therapy Using Atorvastatin Stabilizes Coronary Artery Plaque as Assessed by Multi-detector Row Computed Tomography
}

\author{
Ryoko Mitsutake, Shin-ichiro Miura, Keisuke Okamura, Hideya Niimura, Akira Kawamura \\ and Keijiro Saku
}

\begin{abstract}
A 35-year-old male was diagnosed as angina pectoris and showed severe stenosis with soft plaque in the proximal segment of the left anterior descending (LAD) coronary artery as detected by multi-detector row computed tomography (MDCT). Although percutaneous coronary stent implantation to the LAD lesion was performed, soft plaque remained in the proximal lesion of the stent. Atorvastatin increased the coronary plaque density at the 6-month follow-up MDCT examination, and the low-density lipoprotein cholesterol level fell from 141 to $63 \mathrm{mg} / \mathrm{dl}$ after 6 months. This case may indicate that assessment of the shape or composition of coronary plaque by MDCT is a useful strategy for judging the effects of intensive lipid-lowering therapy using statin.
\end{abstract}

Key words: intensive lipid-lowering therapy, statin, coronary artery plaque, multi-detector row computed tomography

(DOI: 10.2169/internalmedicine.46.6434)

\section{Introduction}

Recent studies have demonstrated that lowering lowdensity lipoprotein cholesterol (LDL-C) levels by statin therapy markedly reduces the risk of coronary artery disease (CAD) and its complications (1). Several invasive studies using intravascular ultrasound (IVUS) have shown that the beneficial effects of statin therapy are involved in the regression or lack of progression of coronary plaque size (2-4).

Recently, multi-detector row computed tomography (MDCT) has become more widely available in many general hospitals, and enables the non-invasive imaging of plaque in the coronary artery, and the accurate assessment of coronary artery stenosis, especially for non-calcified lesions $(5,6)$. We describe here a patient with coronary artery plaque who was treated by intensive lipid-lowering therapy. MDCT was useful for judging the effects of treatment.

\section{Case Report}

A 35-year-old Japanese male was admitted for chest pain on mild exertion. He had no prior history of chest pain, and no notable past history. His father had hypertension and hyperlipidemia. His body mass index was $22 \mathrm{~kg} / \mathrm{m}^{2}$. Blood pressure was 136/70 $\mathrm{mmHg}$, pulse $80 / \mathrm{min}$, regular. Chest $\mathrm{X}$ ray was almost normal (cardiothoracic ratio: 51\%) without pulmonary edema. With regard to the lipid profile, total cholesterol was $208 \mathrm{mg} / \mathrm{dl}$, triglyceride $97 \mathrm{mg} / \mathrm{dl}$, high-density lipoprotein cholesterol $56 \mathrm{mg} / \mathrm{dl}$, and LDL-C $141 \mathrm{mg} / \mathrm{dl}$. His only coronary risk factor was hypercholesterolemia because he had no hypertension, diabetes, smoking, family history (acute myocardial infarction or sudden death) or obesity. An electrocardiogram at rest showed a normal sinus rhythm and no ST change, while an exercise stress electrocardiogram test showed significant ST depression in leads II, III, aVF, and V4 to V6. Therefore, 16-MDCT (Aquilion 16, Toshiba, Tokyo, Japan) was performed for cardiac imaging. The presence of coronary atherosclerotic plaque and significant coro- 
A

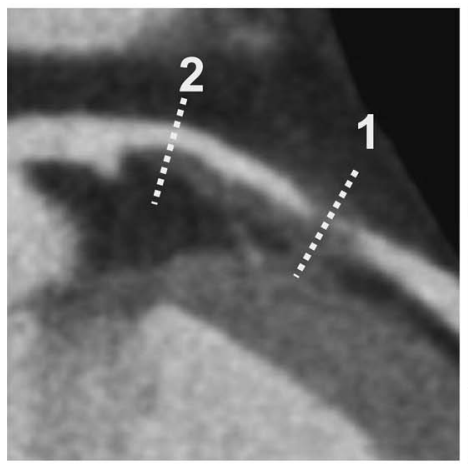

B
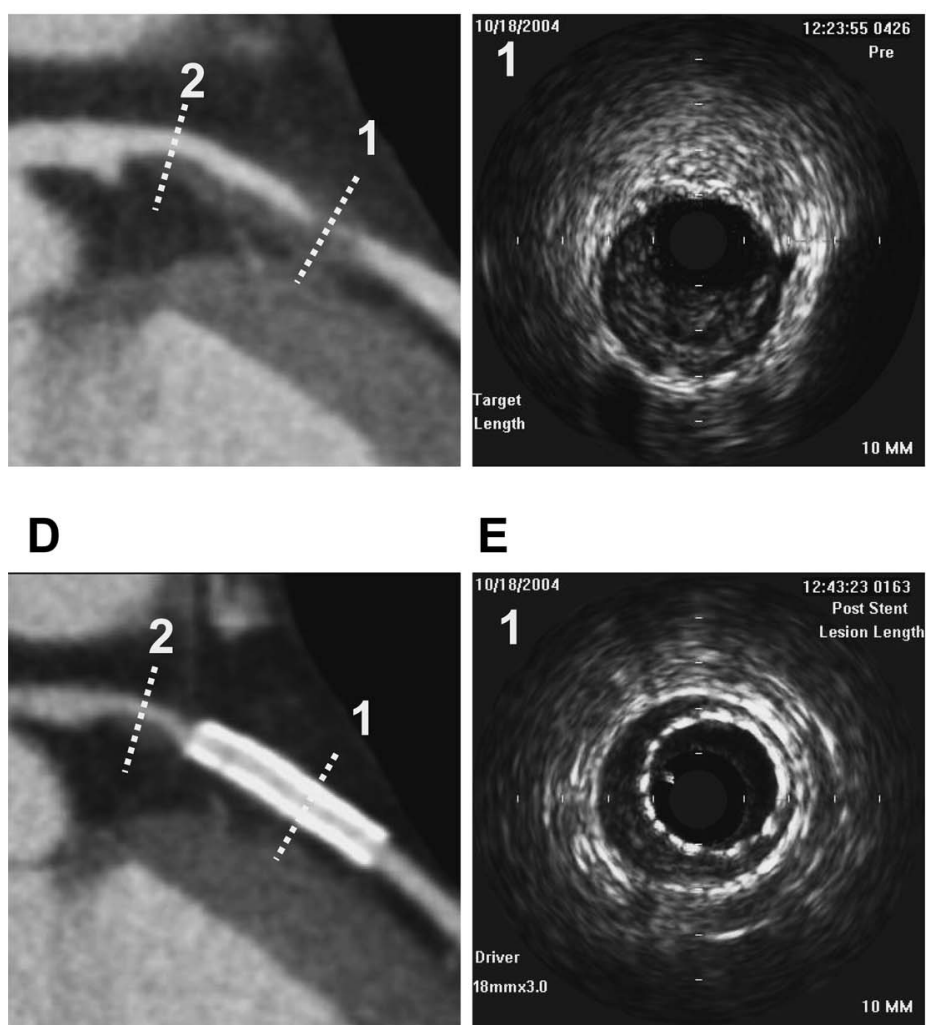

E

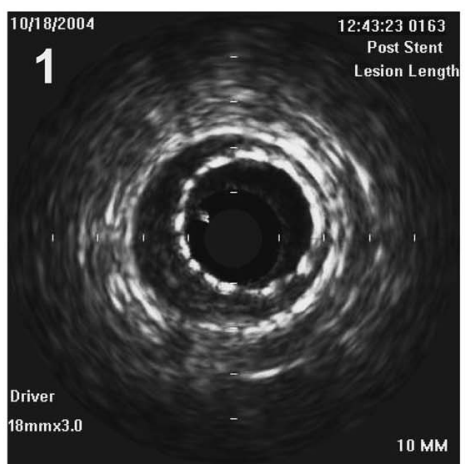

C

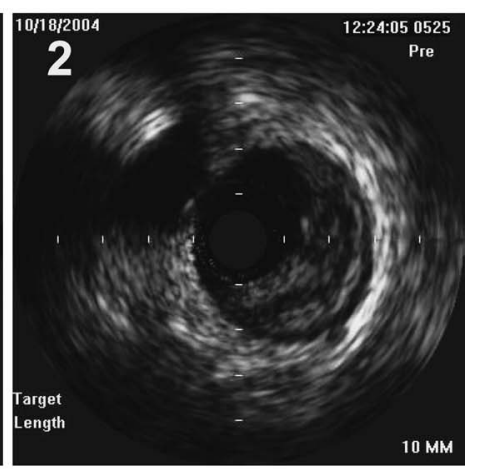

F

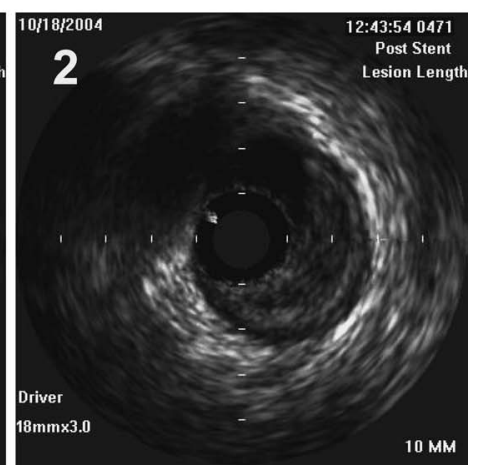

Figure 1. Multi-planar reconstructed multi-detector row computed tomography (MDCT) images at pre- (A) and post- (D) stent implantation and intravascular ultrasound (IVUS) images at pre- (B and $\mathrm{C}$ ) and post- (E and $\mathrm{F})$ stent implantation.

nary artery stenosis was analyzed using a workstation (M 900 Quadra, ZIO SOFT). The results showed severe stenosis with plaque in the proximal left anterior descending artery (LAD), and the plaque extended outward from the vessel wall (positive remodeling) (Figs. $1 \mathrm{AD}$ and 2). The mean density of the plaque was 37.4 hounsfield units (HU) (Fig. 3A). This low-density plaque implied soft plaque, which could cause acute coronary syndrome (ACS). Four days after admission, cardiac catheterization was performed. Coronary angiography also revealed significant stenosis of the proximal LAD (\#6), and coronary stent implantation was performed (Fig. 1). IVUS was also performed before and after stent implantation. Before stent implantation, IVUS images showed severe stenosis with soft plaque that was less echogenic than the surrounding adventitia (Fig. 1ABC). After stent implantation, IVUS showed good stent expansion at the lesion site (Fig. 1D-1 and 1E), and the plaque remained in the proximal side of the stent (Fig. 1D-2 and 1F).

Since he has hypercholesterolemia, atorvastatin $(10 \mathrm{mg} /$ day) was given to stabilize coronary plaque and prevent ACS. Atorvastatin, aspirin (100 mg/day) and ticlopidine (200 mg/day, for only 2 weeks after stent implantation) were administered for 6 months. Six months after beginning statin therapy, the LDL-C level had decreased from 141 to $63 \mathrm{mg} /$ dl. A follow-up MDCT examination was performed at 6 months. The mean density of the plaque was $72.6 \mathrm{HU}$, and thus the plaque density had increased with statin therapy
(Fig. 3B).

\section{Discussion}

The plaque detected by MDCT in the present case showed low density and positive remodeling, which make the plaque more vulnerable to rupture (7-9). Randomized statin trials in patients with ACS and stable CAD have demonstrated that cardiovascular events and coronary restenosis are reduced and cardiovascular survival is optimized when LDL-C is reduced to $<70 \mathrm{mg} / \mathrm{dl}(10-13)$. O'Keefe et al reported that the cumulative randomized trial experience supports LDL-C reductions of $50 \%$ in secondary prevention (14). In the present case, atorvastatin was used to reduce the LDL cholesterol level from 141 to $63 \mathrm{mg} / \mathrm{dl}$ (46\% decrease) as secondary prevention.

Qualitative analysis in an IVUS study in patients who were treated with up to $80 \mathrm{mg}$ of atorvastatin showed an increase in plaque hyperechogenicity that resulted from a change in the plaque composition (15). On the other hand, quantitative analysis in a recent IVUS study showed that significant coronary plaque regression occurred after statin treatment in patients with ACS who were treated with 20 $\mathrm{mg}$ atorvastatin for 6 months $(7,8)$. In this case, there was an increase in plaque density without a change in plaque size after treatment with atorvastatin at $10 \mathrm{mg}$. The dose of atorvastatin was less than that in the ESTABLISH study (2), 

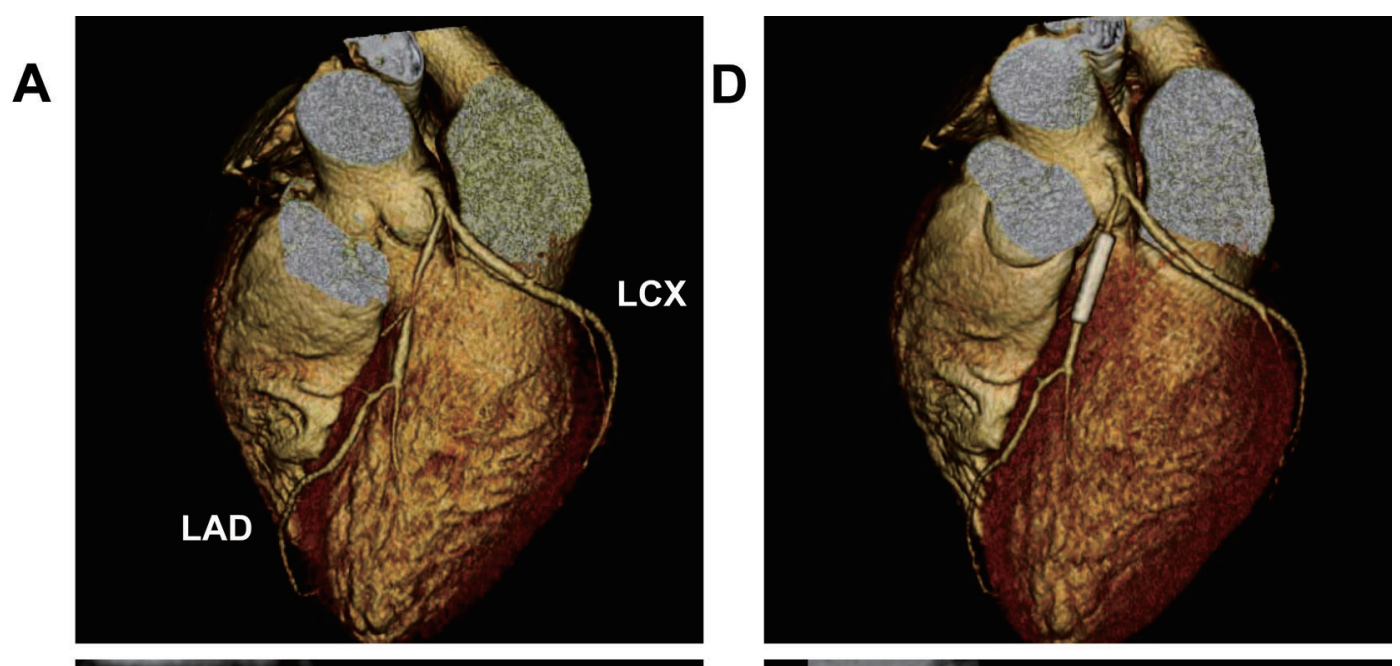

B

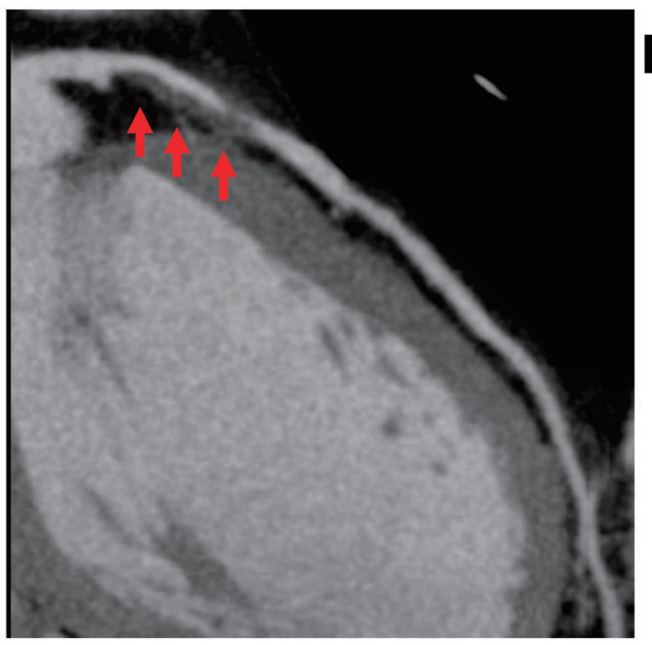

E
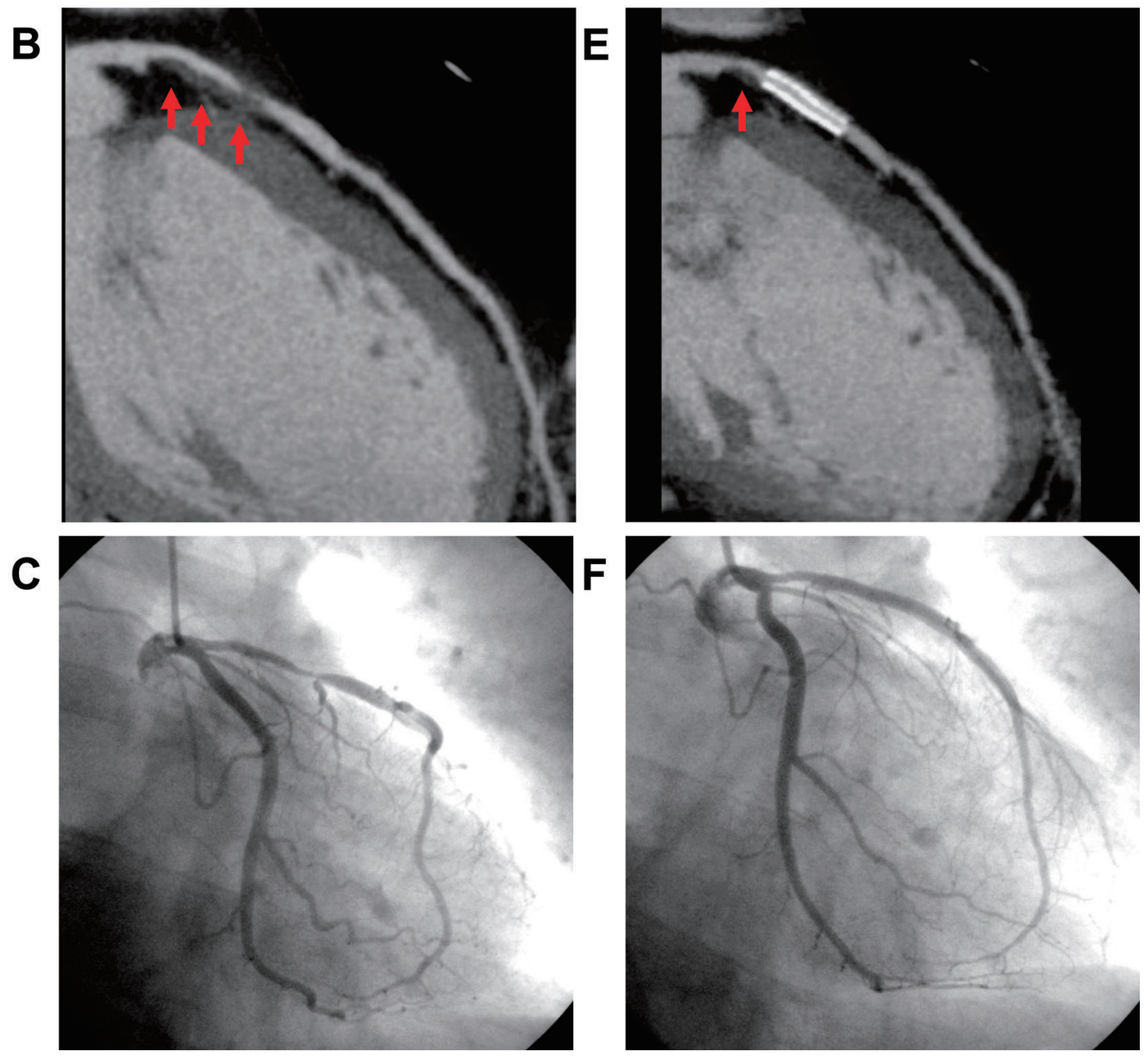

Figure 2. Multi-detector row computed tomography (MDCT) was performed at pre-stent implantation (A and B) and at a six-month follow-up (D and E). A and D show VR, and B and E show multi-planar reconstructed images. CAG results are shown in $\mathrm{C}$ (at pre-stent implantation) and $\mathrm{F}$ (at a six-month follow-up). Severe stenosis (A, B and C) and plaque with positive remodeling (B) were identified in the proximal left anterior descending artery (LAD). Plaque remained in the proximal part of the LAD (E). Arrows indicate plaque (B and E).

and the duration of atorvastatin administration and the reduction in LDL-C by atorvastatin were considered adequate. Although Achenbach et al reported that cross-sectional vessel areas and plaque size measured by MDCT were closely correlated with those by IVUS (16), the spatial resolution of
MDCT is not presently high enough to accurately trace the shape of the vessel wall and the plaque. However, plaque size or positive remodeling was unchanged in this patient, and this may have been due to the inadequate resolution of MDCT. 


\begin{tabular}{|c|c|c|}
\hline & stent implantation & Six-month follow-up \\
\hline Plaque density & $37.2 \mathrm{HU}$ & $72.6 \mathrm{HU}$ \\
\hline Plaque size & $0.13 \mathrm{~cm}^{2}$ & $0.12 \mathrm{~cm}^{2}$ \\
\hline Total cholesterol & $208 \mathrm{mg} / \mathrm{dl}$ & $143 \mathrm{mg} / \mathrm{dl}$ \\
\hline Triglyceride & $97 \mathrm{mg} / \mathrm{dl}$ & $67 \mathrm{mg} / \mathrm{dl}$ \\
\hline HDL-cholesterol & $56 \mathrm{mg} / \mathrm{dl}$ & $58 \mathrm{mg} / \mathrm{dl}$ \\
\hline LDL-cholesterol & $141 \mathrm{mg} / \mathrm{dl}$ & $63 \mathrm{mg} / \mathrm{dl}$ \\
\hline C-reactive protein & $0.5 \mathrm{mg} / \mathrm{dl}$ & 0 mg/dl \\
\hline
\end{tabular}

Figure 3. Cross-sectional image in the left anterior descending artery (LAD) by multi-detector row computed tomography (MDCT) and lipid profiles at pre-stent implantation (A) and at a sixmonth follow-up (B). Vessel lumen (arrowhead) and plaque (arrow) were clearly identified. An increase in plaque density was seen without a decrease in size.

The feasibility of using MDCT to evaluate coronary stenosis (17), detect coronary plaque (18) and measure the vessel and plaque area by manual tracing has been reported (16). In a study that compared IVUS and MDCT, Schroeder et al (19) reported that the mean density of soft plaque was $14 \pm 26 \mathrm{HU}(-14$ to $+47 \mathrm{HU})$, that of intermediate plaque was $91 \pm 21 \mathrm{HU}$ (61 to $112 \mathrm{HU}$ ), and that of calcified plaque was $419 \pm 194 \mathrm{HU}(419 \pm 194 \mathrm{HU})$. In this case, MDCT examination before treatment showed a mean plaque density of 37.4 HU, which was classified as ACS-prone soft plaque. At a 6month follow-up MDCT examination, the mean plaque density had increased to $72.6 \mathrm{HU}$, which was classified as intermediate plaque. This result indicated that the plaque composition may have changed, and that soft plaque might have been stabilized. Although the overall accuracy of MDCT for determining plaque morphology (soft, fibrous, fibrocalcific or calcific) was $92 \%$ in a comparison study with IVUS (20), this study lacked a control case without statin administration. Therefore, this study was limited with regard to whether plaque stability was due to statin therapy or the time course of the plaque change.

The shape and composition of plaque may change between before and after stent implantation. Compared with an IVUS study before and after stent implantation, the shape and composition of remaining plaque did not change in this case. In an evaluation of plaque located near a stent, plaque density might be elevated by halation of the stent. However, the influence of stent implantation on plaque density was less likely because slice adjustment was corrected with a side branch.

In conclusion, this case may indicate that intensive lipidlowering therapy using atorvastatin could contribute to the stabilization of coronary soft plaque and secondary prevention. In addition, noninvasive MDCT may be an important diagnostic modality in patients with suspected CAD, and could be valuable for monitoring plaque stabilization in patients who are undergoing intensive lipid-lowering therapy with statins.

\section{References}

1. LaRosa JC, Grundy SM, Waters DD, et al. Intensive lipid lowering with atorvastatin in patients with stable coronary disease. $\mathrm{N}$ Engl J Med 352: 1425-1435, 2005.

2. Okazaki S, Yokoyama T, Miyauchi K, et al. Early statin treatment in patients with acute coronary syndrome: demonstration of the beneficial effect on atherosclerotic lesions by serial volumetric intravascular ultrasound analysis during half a year after coronary event: the ESTABLISH study. Circulation 110: 1061-1068, 2004.

3. Jensen LD, Thayssen P, Pedersen KE, Stender S, Haghfelt T. Re- gression of coronary atherosclerosis by simvastatin: a serial intravascular ultrasound study. Circulation 110: 265-270, 2004.

4. Nissen SE, Tuzcu EM, Schoenhagen P, et al. Effect of intensive compared with moderate lipid-lowering therapy on progression of coronary atherosclerosis: a randomized controlled trial. JAMA 291: 1071-1080, 2004.

5. Rumberger JA, Sheedy PF 3rd, Breen JF, Schwartz RS. Coronary calcium, as determined by electron beam computed tomography, and coronary disease on arteriogram. Effect of patient's sex on 
diagnosis. Circulation 91: 1363-1367, 1995.

6. Mitsutake R, Niimura H, Miura S, et al. Clinical significance of the coronary calcification score by multidetector row computed tomography for the evaluation of coronary stenosis in Japanese patients. Circ J 70: 1122-1127, 2006.

7. Smits PC, Pasterkamp G, de Jaegere PP, et al. Angioscopic complex lesions are predominantly compensatory enlarged: an angioscopy and intracoronary ultrasound study. Cardiovasc Res 41: 458-464, 1999.

8. Schoenhagen P, Ziada KM, Kapadia SR, et al. Extent and direction of arterial remodeling in stable versus unstable coronary syndromes: an intravascular ultrasound study. Circulation 101: 598603, 2000.

9. Pasterkamp G, Schoneveld AH, van der Wal AC, et al. Relation of arterial geometry to luminal narrowing and histologic markers for plaque vulnerability: the remodeling paradox. J Am Coll Cardiol 32: 655-662, 1998.

10. Cannon $\mathrm{CP}$, Braunwald $\mathrm{E}$, McCabe $\mathrm{CH}$, et al. Intensive versus moderate lipid lowering with statins after acute coronary syndromes. N Engl J Med 350: 1495-1504, 2004.

11. Pedersen TR, Faergeman O, Kastelein JJ, et al. High-dose atorvastatin vs usual-dose simva-statin for secondary prevention after myocardial infarction: the IDEAL study: a randomized controlled trial. JAMA 294: 2437-2445, 2005.

12. Baigent C, Keech A, Kearney PM, et al. Efficacy and safety of cholesterol-lowering treatment: prospective meta-analysis of data from 90,056 participants in 14 randomised trials of statins. Lancet 366: 1267-1278, 2005.

13. Iwata A, Miura S, Shirai K, et al. Lower level of low-density lipoprotein cholesterol by statin prevents progression of coronary restenosis after successful stenting in acute myocardial infarction. Intern Med 45: 885-890, 2006.

14. O'Keefe JH Jr, Cordain L, Harris WH, Moe RM, Vogel R. Optimal low-density lipoprotein is 50 to $70 \mathrm{mg} / \mathrm{dl}$ : lower is better and physiologically normal. J Am Coll Cardiol 43: 2142-2146, 2004.

15. Schartl M, Bocksch W, Koschyk DH, et al. Use of intravascular ultrasound to compare effects of different strategies of lipidlowering therapy on plaque volume and composition in patients with coronary artery disease. Circulation 104: 387-392, 2001.

16. Achenbach S, Ropers D, Hoffmann U, et al. Assessment of coronary remodeling in stenotic and nonstenotic coronary atherosclerotic lesions by multidetector spiral computed tomography. J Am Coll Cardiol 43: 842-847, 2004.

17. Sato Y, Kanmatsuse K, Inoue F, et al. Noninvasive coronary artery imaging by multislice spiral computed tomography. Circ J 2: 107111, 2003.

18. Ropers D, Baum U, Pohle K, et al. Detection of coronary artery stenoses with thin-slice multi-detector row spiral computed tomography and multiplanar reconstruction. Circulation 107: 664666, 2003.

19. Schroeder S, Kopp AF, Baumbach A, et al. Noninvasive detection and evaluation of atherosclerotic coronary plaques with multislice computed tomography. J Am Coll Cardiol 37: 1430-1435, 2001.

20. Rasouli ML, Shavelle DM, French WJ, McKay CR, Budoff MJ. Assessment of coronary plaque morphology by contrast-enhanced computed tomographic angiography: comparison with intravascular ultrasound. Coron Artery Dis 4: 359-364, 2006.

(C) 2007 The Japanese Society of Internal Medicine http://www.naika.or.jp/imindex.html 